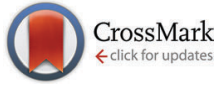

Cite this: Chem. Commun., 2014, 50, 12955

Received 23rd July 2014,

Accepted 5th September 2014

DOI: $10.1039 / c 4 c c 05531 d$

www.rsc.org/chemcomm

\section{Ultrasound-induced transformation of fluorescent organic nanoparticles from a molecular rotor into rhomboidal nanocrystals with enhanced emission $\dagger$}

\author{
Matthias Koenig, ${ }^{a}$ Tomás Torres, ${ }^{b c}$ Vincenzo Barone, ${ }^{d}$ Giuseppe Brancato, ${ }^{\text {d }}$ \\ Dirk M. Guldi ${ }^{\star a}$ and Giovanni Bottari ${ }^{\star b c}$
}

Fluorescent organic nanoparticles (FONs) based on aggregation-induced emission (AIE) are receiving increasing attention owing to their simple preparation, enhanced optical properties, and a wide range of applications. Therefore, finding simple methods to tune the structural and emissive properties of FONs is highly desirable. In this context, we discuss the preparation of highly emissive, amorphous AIE spherical nanoparticles based on a structurally-simple molecular rotor and their sonochemical transformation into rhomboidal nanocrystals. Interestingly, the ultrasound-induced modification of the morphology is accompanied by a remarkable enhancement in the stability and emission of the resulting nanocrystals. Detailed characterization of both spherical and rhomboidal nanoparticles was carried out by means of several microscopic, crystallographic, and spectroscopic techniques as well as quantum mechanical calculations. In a nutshell, this work provides a unique example of the ultrasound-induced switching of morphology, stability, and emission in FONs.

In recent years, the interest in fluorescent organic nanoparticles (FONs) ${ }^{1}$ has been triggered by important advances in their preparation, characterization, and applications in the area of photonic materials, ${ }^{2}$ optoelectronics, ${ }^{3}$ (bio)chemical sensing ${ }^{1 a, 4}$ and in vitro/ in vivo bio-imaging, ${ }^{1,5}$ just to mention a few. Among the plethora of molecular building blocks used for the preparation of FONs, those showing "aggregation-induced emission" (AIE) are particularly interesting and promising. 6 In contrast to planar organic molecules, which present "aggregation-caused quenching", AIE systems are flexible and non-planar. They are usually poorly emissive in solution,

\footnotetext{
${ }^{a}$ Department of Chemistry and Pharmacy and Interdisciplinary Center for Molecular Materials (ICMM), Friedrich-Alexander-Universität Erlangen-Nürnberg, 91058 Erlangen, Germany. E-mail: dirk.guldi@fau.de; Tel: +49-9131-85-27341

${ }^{b}$ Organic Chemistry Department, Universidad Autónoma de Madrid, 28049 Cantoblanco, Spain. E-mail: giovanni.bottari@uam.es; Tel: +34 914972777 ${ }^{c}$ IMDEA-Nanociencia, c/Faraday 9, Campus de Cantoblanco, 28049 Madrid, Spain

${ }^{d}$ Scuola Normale Superiore, Piazza dei Cavalieri 7, I-56126 Pisa, Italy.

E-mail: giuseppe.brancato@sns.it; Fax: +34050 509071

$\dagger$ Electronic supplementary information (ESI) available: Synthetic procedure, spectroscopic, microscopic and crystallographic data for DPAP nanoparticles. See DOI: $10.1039 / \mathrm{c} 4 \mathrm{cc} 05531 \mathrm{~d}$
}

but strongly fluorescent upon aggregation due to restricted intramolecular rotation. ${ }^{7}$ One of the simplest and most successful strategies for the preparation of AIE nanoparticles encompasses on the reprecipitation method. ${ }^{8}$ Implicit is a solvent-exchange process, which mostly produces amorphous nanoparticles. Notably, their size and morphology is tunable by varying experimental conditions including temperature, concentration, aging time, nature and ratio of solvents, etc. However, crystalline FONs are often preferred, owing to their higher rigidity and stability especially in view of biological and optoelectronic applications. ${ }^{9}$

In this work, we focused on the aggregation of an easy-to-prepare molecular rotor, namely 4-(diphenylamino)-phthalonitrile (DPAP), leading to fluorescent, amorphous spherical nanoparticles and, subsequently, even stronger fluorescent rhomboidal nanocrystals. More precisely, a reprecipitation method was employed for the synthesis of AIE DPAP nanoparticles. The latter were subsequently transformed into flat rhomboidal nanocrystals of a high aspect ratio via short ultrasonication. Remarkably, such an unprecedented, ultrasound-induced modification of the nanoparticle morphology increased the stability - from a few hours to months - and the emission - 3-fold - of the resulting nanocrystals. While sonochemical transformations of molecular aggregates have been reported for other systems, ${ }^{10}$ this is the first case, to the best of our knowledge, of such a morphology change in the context of fluorescent organic probes leading to AIE nanocrystals. Therefore, we reckon that such an example is a convenient and promising synthetic strategy for the preparation of FONs. We employed steady-state and time-resolved spectroscopic techniques to gain insights into the photophysical processes. Additionally, we turned to a number of microscopic methods, namely Cryotransmission electron microscopy (Cryo-TEM), atomic force microscopy (AFM) and fluorescence imaging, along with quantum mechanical (QM) calculations. Such a comprehensive approach allowed for a detailed analysis of the structural and emissive features of the fluorescent nanostructures.

DPAP is a fluorescent molecular rotor, whose emission responds sensitively to both solvent polarity and viscosity (Fig. 1a). ${ }^{11}$ On one hand, in apolar or poorly-polar solvents 
a)
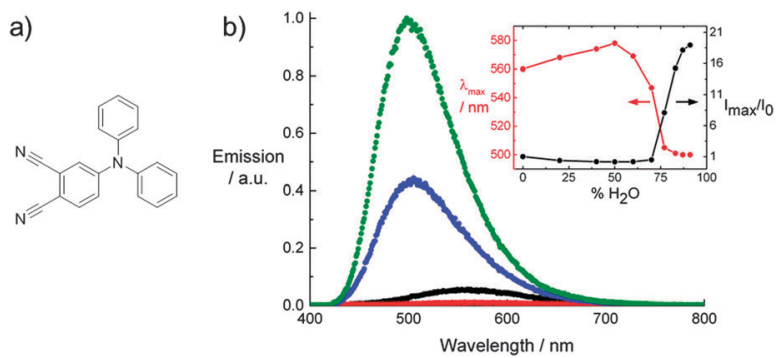

Fig. 1 (a) Molecular structure of DPAP. (b) Fluorescence spectra $\left(\lambda_{\text {exc }}=\right.$ $324 \mathrm{~nm})$ of DPAP $\left(4.5 \times 10^{-5} \mathrm{M}\right)$ in methanol (black) and in water/methanol mixtures $-60 / 40(\mathrm{v} / \mathrm{v})(\mathrm{red}), 77 / 23(\mathrm{v} / \mathrm{v})(\mathrm{blue})$, and 87/13 (v/v) (green). Inset in (b): relative emission intensities (right) and emission maxima (left) of DPAP in the water-methanol mixtures as a function of water content.

DPAP is highly emissive with quantum yields as high as 0.42 . On the other hand, in polar and protic solvents its fluorescence is strongly reduced, with quantum yields as low as $0.0021 \mathrm{in}$, for example, methanol (Fig. 1b). The modus operandi of DPAP implies a significant stabilization of an intramolecular charge transfer (ICT) state and its fast deactivation via non-radiative processes in polar and protic solvents.

Finding methods to guarantee the strong emissive character of DPAP in protic environments would significantly broaden the applicability of this molecular sensor. To this end, the emission properties of DPAP in mixtures of polar and protic solvents, such as water and methanol, in which DPAP presents significant solubility differences, have been carefully investigated. DPAP is extremely soluble in methanol, but highly insoluble in water.

Upon adding up to $70 \%$ of water to a methanol solution of DPAP, the rotor fluorescence is quenched with respect to the emission of DPAP in pure methanol. In turn, such an increase in polarity favors the deactivation via non-radiative pathways. However, when the $70 \%$ water content is surpassed, the emission gradually intensifies reaching a maximum at about $87 \%$ of water (Fig. 1b, inset). Interestingly, a 20-fold DPAP emission increase with a quantum yield of 0.06 , when going from methanol to a $87 / 13(\mathrm{v} / \mathrm{v})$ water/methanol mixture, is accompanied by a blue shift of the emission maximum of $c a .60 \mathrm{~nm}$, that is, from 560 to $500 \mathrm{~nm}$. In contrast, a red shift of the absorption maximum emerges for DPAP from $324 \mathrm{~nm}$ in methanol to $332 \mathrm{~nm}$ in the water-methanol mixture. Along with this, a broad tail reaching almost $600 \mathrm{~nm}$ points to the formation of DPAP aggregates (Fig. S1, ESI $\dagger$ ). Examining dispersions of different DPAP concentrations inferred that AIE sets in above $2 \times 10^{-5} \mathrm{M}$ (Fig. S2, ESI $\dagger$ ).

However, a major drawback of these DPAP aggregates is their low stability as revealed by a significant decrease in emission of the rotor dispersion over time (Fig. S3, ESI $\dagger$ ), accompanied by the formation of a precipitate. In order to stabilize DPAP aggregates, a slightly different procedure for the preparation of the AIE dispersions was employed. In detail, DPAP dispersions were ultrasonicated for 1 minute. Surprisingly, the ultrasound treatment afforded DPAP dispersions which were stable for months - a conclusion that stems from reproducible absorption and emission spectra (Fig. S4 and S5, ESI $\dagger$ ). Moreover, the observed sonication-induced stabilization of the DPAP

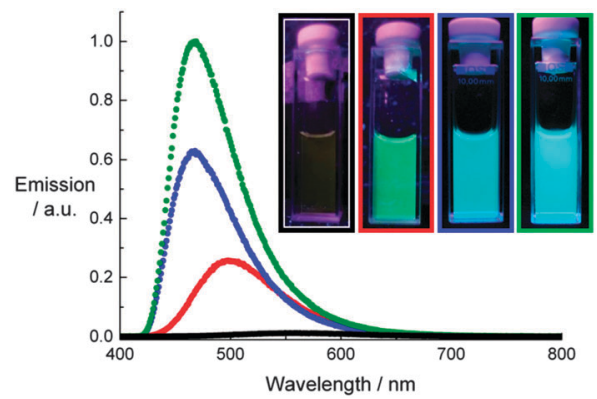

Fig. 2 Fluorescence spectra $\left(\lambda_{\text {exc }}=324 \mathrm{~nm}\right)$ of DPAP $\left(4.5 \times 10^{-5} \mathrm{M}\right)$ in methanol (black) and in 87/13 (v/v) water/methanol mixtures without (red) and with ultrasonication treatment at room temperature (blue) and at $258 \mathrm{~K}$ (green). Inset: photographs of the four samples with a colored box corresponding to the respective emission spectra.

aggregates was accompanied by a 3 -fold emission increase and a quantum yield of 0.13 . At the same time, a blue-shift of the emission maximum from 500 to $470 \mathrm{~nm}$ was noted (Fig. 2). Similarly, emission lifetimes determined for sonicated and unsonicated DPAP dispersions showed a drastic increase of the main component to 8.6 and $12.5 \mathrm{~ns}$, respectively, compared to $0.56 \mathrm{~ns}$ for a methanol solution (Table S1 and Fig. S6, ESI $\dagger$ ).

These findings suggest that the sonochemical treatment gives rise to changes in the rotor organization within the AIE aggregates (vide infra).

Recording the fluorescence features of a frozen, ultrasonicated dispersion of DPAP revealed a further emission increase of ca. 1.6-fold with respect to the fluorescence of the ultrasonicated dispersion at room temperature. Note that the emission features at both temperatures are virtually superimposable (Fig. 2). ${ }^{12}$

Further information about the structural and morphological features of DPAP aggregates were borrowed from dynamic light scattering (DLS), Cryo-TEM and AFM measurements. Firstly, DLS measurements with unsonicated and sonicated DPAP dispersions at $4.5 \times 10^{-5} \mathrm{M}$ showed the presence of particles with hydrodynamic radii of about 80 and $100 \mathrm{~nm}$, respectively (Fig. S7, ESI $\dagger$ ). ${ }^{13}$ Secondly, Cryo-TEM studies with unsonicated DPAP dispersions at $4.5 \times 10^{-5}$ and $2.5 \times 10^{-4} \mathrm{M}$ revealed spherical nanoparticles in the size range of 100-200 nm (Fig. 3a and Fig. S8, ESI $\dagger$ ) which is in line with the DLS results.

An intriguing and unexpected change in the AIE aggregate morphology was observed upon ultrasonication. While ultrasonicated dispersions of DPAP at $4.5 \times 10^{-5} \mathrm{M}$ mainly exhibited irregularly-shaped objects (Fig. 3b), at DPAP concentrations of $2.5 \times 10^{-4} \mathrm{M}$ the formation of large, flat rhomboidal nanostructures on the micro- and sub-micrometer scale prevailed (Fig. 3c). ${ }^{14}$ Analyses carried out on some laterally-positioned nanoparticles revealed that they have an average thickness of ca. 30-35 $\mathrm{nm}$ and that they are composed of several stacked layers (Fig. 3c inset and Fig. S9b, ESI $\dagger$ ). ${ }^{15,16}$

To better characterize these nanostructures, selected-area electron diffraction (SAED) experiments were performed on both face-on and laterally-positioned rhomboidal nanoparticles. The obtained highly symmetrical electron diffraction patterns supported the crystalline nature of the sonochemically-synthesized nanoparticles. A detailed 

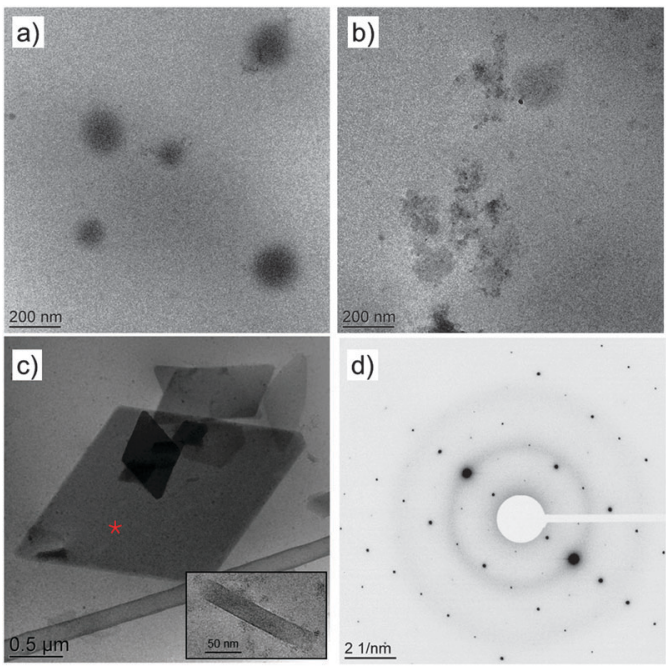

d)

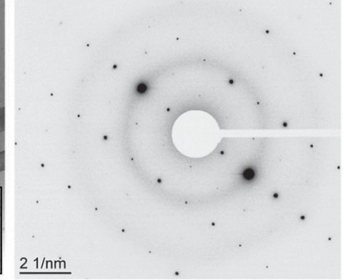

Fig. 3 Cryo-TEM images of a 87/13 (v/v) water/methanol dispersion of DPAP without (a) and with (b, c) ultrasonication treatment. In $(a, b)$ the DPAP concentration is $4.5 \times 10^{-5} \mathrm{M}$, whereas in (c) it is $2.5 \times 10^{-4} \mathrm{M}$. Inset in (c): side-view of a nanocrystal. (d) SAED pattern recorded on one of the nanocrystals. The five-point, red star in (c) indicates the center of the radiation beam in the SAED experiment.

analysis of the face-on diffraction pattern afforded 7.4 and 7.6 $\AA$ as the two unit cell dimensions (Fig. 3d). In addition, a lattice distance of $13.0 \AA$ was obtained from a laterally-positioned nanocrystal (Fig. S9b, ESI, $\dagger$ inset). As such, these values are in sound agreement with the unit cell dimensions of DPAP found in X-ray diffraction studies on a single crystal ( $a=8.1851 \AA$, $b=$ $8.5344 \AA$ and $c=13.5521 \AA$ ) (Fig. S10, ESI $\dagger$ ). ${ }^{12}$ At this point we hypothesize that the arrangement of DPAPs within the nanocrystals must be similar to that seen in the single crystal. In line with this hypothesis is the finding that the two internal angles in most rhomboidal nanocrystals are comprised between $114^{\circ}$ $118^{\circ}$ and $62^{\circ}-65^{\circ}$ (Fig. S11 and S12, ESI $\dagger$ ). These are in close agreement with the internal angles formed by the $a$ and $b$ axes in the single-crystal DPAP unit cell $\left(\gamma=114.976^{\circ}\right.$ and $\left.\phi=65.024^{\circ}\right)$ (Fig. S10, ESI $\dagger$ ). To the best of our knowledge, this is a rare example of an organic (nano)crystal which grows maintaining some of its unit cell crystal lattice parameters. ${ }^{17}$ On a further note, despite the different morphologies in the ultrasonicated DPAP nanoparticle dispersions, that is, at low and high concentrations, the emission was very similar (Fig. S13, ESI + ). This suggests that the molecular arrangement in the crystalline nanostructures is also present in the irregularly-shaped materials.

Furthermore, the observed blue-shift of the DPAP emission, when going from solution $(560 \mathrm{~nm})$ to the nanocrystals $\left(470 \mathrm{~nm}\right.$ ), with $\Delta \lambda_{\exp }=90 \mathrm{~nm}$, has been reproduced by QM calculations with $\Delta \lambda_{\text {calc }}=80-100 \mathrm{~nm}$. These are based on an optimized geometry of the rotor in solution and in the X-ray crystal structure. In general, the DPAP emission is very sensitive to the dihedral angle $(\theta)$ between the phthalonitrile unit and the amino moiety (Fig. S14a, ESI $\dagger$ ). ${ }^{12}$ While in solution, the dihedral angle is, on average, around $25^{\circ}$, the resolved crystal structure is more planar with an angle of about $12^{\circ}$ (Fig. S14b, ESI $\dagger$ ). Consequently, and along with the possible formation of excitonic species in the nanocrystals, a blue shift of the computed emission is expected. It is worth noting that in the resolved X-ray crystal structure, DPAP molecules are tightly packed along the [001] crystallographic plane, corresponding to the two short unit-cell dimensions $(a \approx b<c)$. This is due to $\pi-\pi$ stacking (Fig. S10b, ESI $\dagger$ ) and dipole-dipole interactions (Fig. S10a, ESI $\dagger$ ). The intermolecular interactions along the $c$ axis, which are mediated by crystallographic solvent molecules, do appear weaker due to van der Waals interactions. This provides a possible explanation for the quasi 2-dimensional growth of DPAP rhomboidal nanocrystals. Indeed, ultrasonication provides the necessary activation energy to trigger the transformation of the amorphous DPAP particles, which are likely the kinetically more favourable assemblies, into the thermodynamically more stable nanocrystals.

Moreover, AFM studies were carried out with the nanocrystals by drop-casting a few $\mu \mathrm{l}$ of a DPAP dispersion at $2.5 \times 10^{-4}$ $M$ on a silicon surface (Fig. 4a). These studies showed the presence of rhomboidal nanostructures, which strongly resemble in size and shape those observed in the Cryo-TEM experiments. The height of these nanoparticles, as determined by AFM, falls between 30 and $40 \mathrm{~nm}$, which is in close agreement with the values from the Cryo-TEM experiments (Fig. 4a, inset).

Fluorescence imaging studies $\left(\lambda_{\mathrm{exc}}=405 \mathrm{~nm}\right)$ have also been performed with DPAP dispersions at $2.5 \times 10^{-4} \mathrm{M}$ by casting a few $\mu \mathrm{l}$ of the rotor dispersion between two glass slides. Here, the presence of highly-fluorescent nanoparticles (Fig. 4b, inset) with an emission maximizing at $470 \mathrm{~nm}$ (Fig. 4b) was corroborated. This fluorescence matches reasonably well with the steady-state emission recorded for the same DPAP dispersion. Both AFM and fluorescence imaging show that such crystalline nanostructures, formed by sonochemical treatment in a water-rich environment, are shape-persistent and mechanically robust. Moreover, they are easily transferable onto different surfaces, keeping their structural integrity and emissive features.

In conclusion, we have presented here the facile synthesis of highly-fluorescent, amorphous spherical nanoparticles from a structurally-simple molecular rotor and their sonochemical-induced transformation into high aspect ratio, rhomboidal nanocrystals. Interestingly, such a morphological change is accompanied
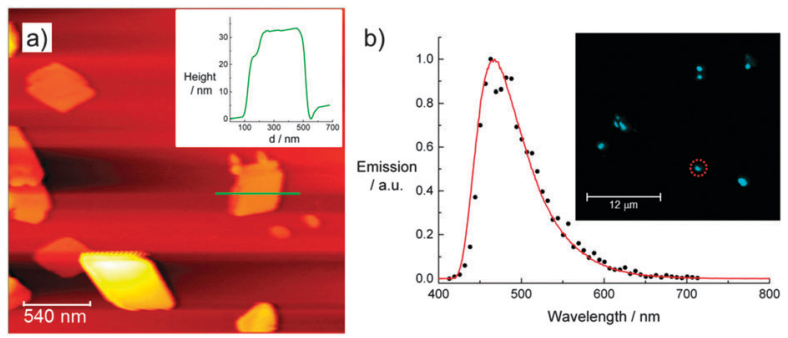

Fig. 4 (a) AFM topographic image of a 87/13 (v/v) water/methanol dispersion of DPAP $\left(2.5 \times 10^{-4} \mathrm{M}\right)$ drop-casted onto a silicon surface. Inset: AFM topographic profile of the green line in (a). (b) Emission spectra of an 87/13 $(\mathrm{v} / \mathrm{v})$ water/methanol dispersion of DPAP $\left(4.5 \times 10^{-5} \mathrm{M}\right)$ (red line, $\lambda_{\mathrm{exc}}=$ $324 \mathrm{~nm}$ ) and of a single DPAP nanocrystal (black dots, $\lambda_{\text {exc }}=405 \mathrm{~nm}$ ). Inset: fluorescence microscopic image of an 87/13 (v/v) water/methanol dispersion of DPAP $\left(2.5 \times 10^{-4} \mathrm{M}\right)$ between two glass slides. The dashed red circle indicates the nanocrystal selected for the emission experiment. 
by a significant modification in the stability and emission of the AIE nanoparticles. Whereas the use of ultrasonication as a means to produce crystalline organic particles has only been rarely reported in the past, ${ }^{18}$ this is the first example, in which such a stimulus serves as a convenient tool to switch morphology, stability, and emission of FONs. This opens the way for the development of novel fluorescent materials with tunable properties.

Financial support from Spanish Ministry of Economy and Competitivity, MICINN (CTQ-2011-24187/BQU), MIUR through FIRB program (contract no. RBFR10DAK6), ERC Advanced Grant 2012 (number 320951), and Bavarian Initiative "Solar Technologies Go Hybrid"' is kindly acknowledged.

\section{Notes and references}

1 (a) A. Kaeser and A. P. H. J. Schenning, Adv. Mater., 2010, 22, 2985-2997; (b) S. Fery-Forgues, Nanoscale, 2013, 5, 8428-8442.

2 (a) A. Patra, Ch. G. Chandaluri and T. P. Radhakrishnan, Nanoscale, 2012, 4, 343-359; (b) C. Cordovilla and T. M. Swager, J. Am. Chem. Soc., 2012, 134, 6932-6935; (c) W. Yao and Y. S. Zhao, Nanoscale, 2014, 6, 3467-3473.

3 (a) B. K. An, S. K. Kwon and S. Y. Park, Angew. Chem., Int. Ed., 2007, 46, 1978-1982; (b) Y. S. Zhao, H. Fu, A. Peng, Y. Ma, D. Xiao and J. Yao, Adv. Mater., 2008, 20, 2859-2876; (c) Y. S. Zhao, H. Fu, A. Peng, Y. Ma, Q. Liao and J. Yao, Acc. Chem. Res., 2010, 3, 409-418; (d) J. Xiao, X. Xiao, Y. Zhao, B. Wu, Z. Liu, X. Zhang, S. Wang, X. Zhao, L. Liu and L. Jiang, Nanoscale, 2013, 5, 5420-5425. 4 (a) H. Li and H. Yan, J. Phys. Chem. C, 2009, 113, 7526-7530; (b) K. Petkau, A. Kaeser, I. Fischer, L. Brunsveld and A. P. H. J. Schenning, J. Am. Chem. Soc., 2011, 133, 17063-17071.

5 (a) Y. Yu, C. Feng, Y. Hong, J. Liu, S. Chen, K. M. Ng, K. Q. Luo and B. Z. Tang, Adv. Mater., 2011, 23, 3298-3302; (b) J. Mérian, J. Gravier, F. Navarro and I. Texier, Molecules, 2012, 17, 5564-5591; (c) A. Jana, K. S. P. Devi, T. K. Maiti and N. D. P. Singh, J. Am. Chem. Soc., 2012, 134, 7656-7659; (d) Y. Li, T. Liu, H. Liu, M.-Z. Tian and Y. Li, Acc. Chem. Res., 2014, 47, 1186-1198; (e) L. Feng, C. Zhu, H. Yuan, L. Liu, F. Lv and S. Wang, Chem. Soc. Rev., 2013, 42, 6620-6633; $(f)$ L. Feng, L. Liu, F. Lv, G. C. Bazan and S. Wang, Adv. Mater., 2014, 26, 3926-3930.
6 (a) J. Luo, Z. Xie, J. W. Y. Lam, L. Cheng, H. Chen, C. Qiu, H. S. Kwok, X. Zhan, Y. Liu, D. Zhu and B. Z. Tang, Chem. Commun., 2001, 1740-1741; (b) Y. Hong, J. W. Y. Lama and B. Z. Tang, Chem. Soc. Rev., 2011, 40, 5361-5388; (c) R. Hu, N. L. C. Leung and B. Z. Tang, Chem. Soc. Rev., 2014, 43, 4494-4562.

7 Y. Hong, J. W. Y. Lama and B. Z. Tang, Chem. Commun., 2009, 4332-4353.

8 H. Nakanishi and H. Oikawa, Reprecipitation method for organic nanocrystals, in Single Organic Nanoparticles, ed. H. Masuhara, H. Nakanishi and K. Sasaki, Springer-Verlag, Berlin, 2003, pp. 17-31.

9 (a) K. Baba, H. Kasai, K. Nishida and H. Nakanishi, Organic nanocrystals for nanomedicine and biophotonics, in Nanocrystals, ed. Y. Masuda, Sciyo, Rijeka, 2010, pp. 311-326; (b) C. Philippot, F. Dubois, M. Maurin, B. Boury, A. Prat and A. Ibanez, J. Mater. Chem., 2012, 22, 11370-11378.

10 (a) N. Chandrasekhar and R. Chandrasekar, Angew. Chem., Int. Ed., 2012, 51, 3556-3561; (b) D. Ke, C. Zhan, A. D. Q. Li and J. Yao, Angew. Chem., Int. Ed., 2011, 50, 3715-3719; (c) J. M. Malicka, A. Sandeep, F. Monti, E. Bandini, M. Gazzano, C. Ranjith, V. K. Praveen, A. Ajayaghosh and N. Armaroli, Chem. - Eur. J., 2013, 19, 12991-13001.

11 M. Koenig, G. Bottari, G. Brancato, V. Barone, D. M. Guldi and T. Torres, Chem. Sci., 2013, 4, 2502-2511.

12 Considering the ICT nature of the DPAP emission, we ascribe this effect to decreasing non-radiative charge recombination processes upon lowering the temperature.

13 At DPAP concentrations of $2.5 \times 10^{-4} \mathrm{M}$ a larger hydrodynamic radius of $110 \mathrm{~nm}$ for the sonicated dispersions was noted (Fig. S7, ESI $\dagger$ ).

14 Cryo-TEM experiments on an ultrasonicated DPAP dispersion at intermediate concentration $\left(1 \times 10^{-4} \mathrm{M}\right)$ feature the presence of both irregularly-shaped material and nanocrystals (Fig. S7a, ESI $\dagger$ ).

15 Extending the ultrasonication time to $10 \mathrm{~min}$ triggered the formation of rhomboidal nanoparticles with increased sizes (Fig. S9c and $\mathrm{d}, \mathrm{ESI} \dagger$ ).

16 Only one example of rhomboidal nanostructures from a purely organic compound has been reported (M. Sathish, K. Miyazawa, J. P. Hill and K. Ariga, J. Am. Chem. Soc., 2009, 131, 6372-6373).

17 X. Zhang, C. Dong, J. A. Zapien, S. Ismathullakhan, Z. Kang, J. Jie, X. Zhang, J. C. Chang, C. S. Lee and S. T. Lee, Angew. Chem., Int. Ed., 2009, 48, 9121-9123.

18 (a) Y. S. Zhao, W. Yang and J. Yao, Phys. Chem. Chem. Phys., 2006, 8, 3300-3303; (b) J. R. G. Sander, D. K. Bucar, J. Baltrusaitis and L. R. MacGillivray, J. Am. Chem. Soc., 2012, 134, 6900-6903. 